\title{
CONF-901035--8
}

Note: This is a preprint of a paper subrnitted for publication. Contents of this paper should not be quoted or referred to without permission of the author(s).

CONF-901035--8

DE91 001277

To be presented at the 37th Annual American Vacuum Society Symposium and Topical Conference, Toronto, Canada, October 8-12, 1990, and to he published in Journal of Vacuum Science and Technology.

\section{DEFECT AND IMPURITY EFFECTS ON THE INITIAL GROWTH OF Ag ON Si(111)}

\author{
J.-K. Zuo and J. F. Wendelken
}

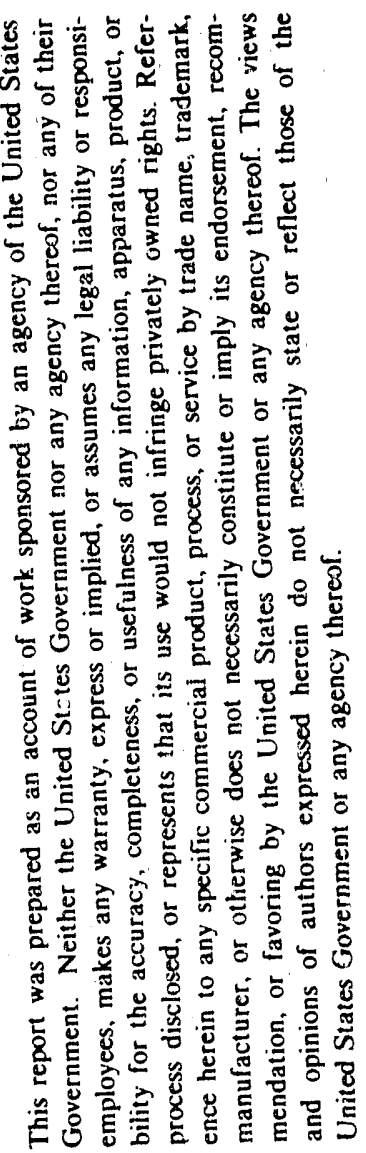

\begin{abstract}
"The submitted manuscript has been authored by contractor of the U.S. Govemment under contract ito. DE. AC05.840R21400. Accordingly, the U.S. Government retains a nonexclusive, royalty-free license to publish or reproduce the published form of this contribution, or wlow others to do so, for U.S. Government purposes:"
\end{abstract}

\author{
SOLID STA'TE DIVISION \\ OAK RIDGE NATIONAL LABORATORY \\ Operated by \\ MARTIN MARIETTA ENERGY SYSTEMS, INC. \\ under \\ Contract No. DE-AC05-84OR21400 \\ with the \\ U.S. DEPARTMENT OF ENERGY \\ Oak Ridge, Tennessee 37831-6024
}

October 1990

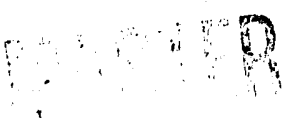




\title{
Defect and Impurity Effects On the initial Growth of Ag On Si(111)
}

\author{
J.-K. Zuo and J. F. Wendelken \\ Solid State Division, Oak Ridge National Laboratory, Oak Ridge, TN 37831
}

\begin{abstract}
Step and impurity effects on the initial growth of a thin film have been demonstrated in the $(\sqrt{3} \times \sqrt{3}) \mathrm{R} 30^{\circ}$ domain growth of $\mathrm{Ag}$ on $\mathrm{Si}(111)$ using high angular resolution LEED. Anisotropy in the $\sqrt{3}$ domain shape and growth during deposition are found on the stepped $S i(111)$ with the preferential growth along the step edge direction. The $\sqrt{3}$ superlattice grows with coverage principally by domain coalescence at the temperature $\mathrm{T} \sim 450^{\circ} \mathrm{C}$ and is self-similar at different coverages (scaling) as observed on a flat $\mathrm{Si}(111)$. The size distribution is shown to follow a Gamma distribution by a simple model calculation. A dramatic change in the growth mechanism is observed when oxygen impurities ( $\leq 0.02 \mathrm{ML})$ appear. The $\sqrt{3}$ domains in the presence of impurities grow with coverage more randomly and isotropically in contrast with the step edge effects and coalescence is inhibited. As a result, the $\sqrt{3}$ superlattice stays in a microdomain morphology without long range order.
\end{abstract}




\section{Introduction}

Defects and impurities on a substrate surface often play important roles in the initial stage of epitaxial growth. ${ }^{1-4}$ Since adatoms which adsorb at a step defect generally have a larger adsorption energy compared to those which adsorb on a terrace, the steps will generally be preferred sites for adsorption and nucleation. Also, in one-dimensional (1D) migration along a step the probability for an adatom or a cluster to meet another adatom or a cluster is very high. Therefore, the overlayer is expected to grow preferentially along a step edge direction. For randomly distributed impurities, the effects may be in opposition to the step effects. The impurities may attract the adatoms and become nucleation centers in competition with the steps, and they will make the overlayer nucleate and grow more randomly. Further, impurities will trap the domain boundaries, keep the domains from coalescing, and finally break the long-range order of the deposited film. In addition to these effects, impurities may also change some basic growth properties such as the growth law and scaling, which has been observed previously. ${ }^{3}$

In the present paper, we demonstrate these step and impurity effects in the initial growth with $\mathrm{Ag} / \mathrm{Si}(111)(\sqrt{3} \times \sqrt{3}) \mathrm{R} 30^{\circ}$ as a model system. This system has been extensively studied by almost every surface analysis technique..$^{5-10}$ Most work has focused on the determination of atomic geometry of the $\sqrt{3}$ structure, with little attention paid to the $\sqrt{3}$ domain growth. In a separate paper, ${ }^{11}$ we reported substrate temperature effects on the $\sqrt{3}$ domain growth as a function of $\mathrm{Ag}$ coverage, where the $\sqrt{3}$ structure was found to grow by domain coalescence at high temperature and by small, randomly nucleated domains at low teriperature. Also, coverage dependent scaling was observed due to domain coalescence and the growth law was extracted at different temperatures. Here, we report our studies for the step difect and oxygen impurity effects on the $\sqrt{3}$. domain growth using a high angular resolution LEED (HRLEED) system. By analyzing the LEED angular profile associated with the $\sqrt{3}$ structure, we are able to determine the $\sqrt{3}$ domain growth mechanism at the initial stage of $\mathrm{Ag}$ deposition on $\mathrm{Si}(111)$ in the presence of steps and impurities. 


\section{Experimental}

The experiments were performed in a UHV chamber containing HRLEED, Auger Electron Spectroscopy (AES) with a double-pass cylindrical mirror analyzer, an Ag evaporation source, and a gas handling system providing various high purity $(6 \mathrm{~N})$ gases. Pressures were routinely in the $10^{-11}$ Torr range. The HRLEED (or Spot-Profile-Analysis LEED ${ }^{12}$ ) is controlled by a personal computer and has a spatial resolution $\leq 6 \times 10^{-3} \AA^{-1}$ in $\mathrm{k}$-space. A good signal-to-noise ratio is obtained by using a channeltron detector. These features allow us to record the angular profile of any diffraction beam accurately and quickly, so that the evolution of the ordered domains can be determined.

The Si samples with size $\sim 0.9 \times 0.9 \mathrm{~cm}^{2}$ were cut from a n-type Si(111) wafer. The misorientation from (111) was less than $0.2^{\circ}$. The sample was mounted in a Mo housing in which the sample could be heated or cooled below room temperature. Temperature was measured by a W5\%Re-W26\%Re thermocouple in contact with a comer of the sample. The Si(111) sample was first annealed at $\sim 1200^{\circ} \mathrm{C}$ to remove the oxide layer and carbon impurities. Later, to desorb $\mathrm{Ag}$ overlayers and oxygen impurities from the substrate and restore the clean $\operatorname{Si}(111) 7 \times 7$ surface, the sample usually was annealed at $\sim 900-1000^{\circ} \mathrm{C}$. After the annealing, no contamination was detectable with AES and the clean $\operatorname{Si}(111)$ surface exhibited a sharp $7 \times 7$ LEED pattern. Ag atoms were evaporated at a fixed rate of $\sim 0.15 \mathrm{ML} / \mathrm{min}$ from a pure $(5 \mathrm{~N}) \mathrm{Ag}$ foil heated by electron bombardment from the backside. We found that this evaporation method can produce a contamination free Ag deposition within our AES detection limit. Oxygen impurities and $\mathrm{Ag}$ coverage were estimated using AES and the total intensity measurement of a $\sqrt{3}$ superlattice LEED beam as described in detail elsewhere. ${ }^{11}$

\section{Step Effects On the $\sqrt{3}$ Domain Growth}

The step density on the Si(111) surface was characterized by measuring the FWHMs of both the $(00)$ and $(1 / 70)$ beams. The FWHM of the $(10)$ beam at an out-of-phase condition, e.g. an incident electron energy $E=78 \mathrm{eV}$, is inversely proportional to the average terrace width and the 
FWHM of the $(1 / 70)$ beam is inversely proportional to the diameter of the average $7 \times 7$ ordered region which was found to be equal to the average terrace sizc. For the "flat" $\mathrm{Si}(111)$ surface, the average terrace width is estimated to be $\geq 1000 \AA$ in all directions, which is our instrument resolution limit. The step defects were created by heating the sample to $\sim 1200-1300{ }^{\circ} \mathrm{C}$ many times. ${ }^{13}$. After this heat treatment, we estimate that the average terrace width is sti'l $\sim 1000 \AA$ along the [211] (step edge) direction but is reduced to $\sim 550 \AA$ along the [0111] direction. At the same time mosaic structures with small mosaic angles were created in the [011] direction during the heat treatment.

When a submonolayer of $\mathrm{Ag}$ is deposited on the $\mathrm{Si}(111) 7 \times 7$ surface above $-200{ }^{\circ} \mathrm{C}$, a $\sqrt{3}$ superlattice forms which is detectable at less than $0.1 \mathrm{ML}$. In our temperature dependent study, ${ }^{11}$ we have learned that with increasing coverage, the $\sqrt{3}$ superlattice grows more perfectly at higher substrate temperature than at lower temperature. Thus, to see the step and impurity effects, it is butter to choose a high temperature $\left(\mathrm{T} \sim 450^{\circ} \mathrm{C}\right)$ as the deposition condition. In our measurements, we have collected the angular profiles of the $(1 / 31 / 3)$ beam as a function of $\mathrm{Ag}$ coverage $(\theta)$ since the angular profile of any superlattice diffraction beam depends solely on the domain size distribution. The depositions were performed at $\mathrm{T}=450^{\circ} \mathrm{C}$ and the LEED measurements were done after the sample cooled to near room temperature. The domain morphology at high deposition temperature may be frozen out when the sample is cooled to room temperature. Figure 1(a) and (b) show the full width at the half maximum (FWHM) of the $(1 / 31 / 3)$ beam vs Ag coverage scanned in the [1̄12] and [2̄11] directions, respectively, for the flat and stepped Si(111) surfaces as well as for two different oxygen impurity doped cases. The oxygen doped cases will be discussed in the next section. We did not measure the angular profile in the [011] direction which is perpendicular to the step edge along the [ $\overline{2} 11]$ direction, but instead measured that in the [112] which is $30^{\circ}$ from the $[0 \overline{1} 1]$. Since the FWHM of any $\sqrt{3}$ superlattice beam is inversely proportional to average domain size in a specific direction, from Fig. 1 we can see that in the [1]12] the $\sqrt{3}$ domain size on the stepped Si(111) becomes smaller than that (dashed curves) on the flat one after $\theta \sim 0.5 \mathrm{ML}$, but in the [211] direction the situation is just the opposite, i.e., the domain 
size on the stepped surface is much larger for -ll coverages. Also, the domain size in the step edge direction is already quite big even at low coverage as indicated by the narrow FWHM. This tells us that on the stepped surface the Ag atoms may be easily captured at steps and the $\sqrt{3}$ domains preferentially grow along the step edge direction. More interestingly, we found that the ratio of the FWHM in the [ $\overline{2} 11]$ to that in the [11 2$]$ does not change with coverage but changes when steps appear. For the flat $\mathrm{Si}(111)$, this ratio (after removing the instrument response width) is $\sim 0.90 \pm$ 0.03 indicating a nearly round domain shape, and it changes to $\sim 0.56 \pm 0.02$ for the stepped surface on which the domains are elongated along the step edge. The domain shapes are determined by several factors. The domain-domain interaction promotes round domains, but the domain-step interaction forces the domains to elongate in the step edge direction. Thus, the invariant domain shape at different coverages on either the flat or stepped surface should be a result of the balance of all these interactions.

This anisotropic growth on the stepped surface is also reflected in the growth law which describes the average domain size as a function of coverage. In Fig. 2(a) and (b) we have plotted $1 /$ FWHM of the $(1 / 31 / 3)$ beam vs $\theta$ in a $\ln$-In scale for the flat and stepped $\mathrm{Si}(111)$ surfaces, where the instrument response width has been removed. As seen in the figures, a power growth law can be established via

$$
\overline{\mathrm{N}} \propto \frac{1}{\mathrm{FWHM}} \propto \theta^{\mathrm{n}} .
$$

For the flat surface, the growth exponent $n$ is fit to be 0.35 and $0.36 \pm 0.02$ in the [ $\overline{2} 11]$ and [ $\overline{1} 12]$ directions, respectively. The similar $\mathrm{n}$ indicates an isotropic growth on the flat surface. However, $\mathrm{n}$ has different values of $0.42 \pm 0.03$ and $0.27 \pm 0.02$ in the [ $[\overline{2} 11]$ and [1 $1 \overline{1} 2]$, respectively, for the stepped surface. As compared with the flat $\mathrm{Si}(111)$ case, the fact that $\mathrm{n}$ is enhanced along the step edge and lowered in the [112] implies that the preferential growth along the step edge is at the expense of the growth in other directions. 
In addition to the above information, it is frequently desirable to know the domain size distribution as a function of coverage at a fixed deposition rate and substrate temperature to gain insight into additional details of the growth mechanism. The domain size distribution can in principle be extracted from the angular profile as long as an appropriate mndel can be established. The model used here assumes that the positions of domain boundaries are randomly distributed so that the interference between domains is negligible. ${ }^{14}$. The intensity of any superlattice beam are then the sum of the intensities diffracted from individual domains, ${ }^{14}$ i.e.,

$$
I(\mathbf{S}, \theta)=\sum_{N=1}^{\infty} P(N, \theta) \frac{\sin ^{2} \frac{N}{2}\left(S_{\|} \cdot \mathbf{a}\right)}{\sin ^{2} \frac{1}{2}\left(S_{\|} \cdot \mathbf{a}\right)}
$$

where the weight $P(N, \theta)$ is the size distribution function describing the probability of finding a domain with $\mathrm{N}$ lattice spacings at a coverage $\theta, \mathbf{S}_{\|}$and a are the momentum transfer parallel to the surface and the lattice constant $(6.65 \AA)$ of the $\sqrt{3}$ structure, respectively. This assumption should hold well for a system with the ground state degeneracy $\mathrm{Q}>2$ because the larger the $\mathrm{Q}$, the more complicated and random domain boundary structure could be. The $Q$ for the $\sqrt{3}$ structure of the $\mathrm{Ag} / \mathrm{Si}(111)$ with the honeycomb arrangement ${ }^{5}$ is larger than 3 , therefore the model should be suitable for our case, especially for the impurity doped cases. For cormparison with the experiment, a distribution function $P(N, \theta)$ can be chosen and $I(S, \theta)$ evaluated using Eq. (2). $I(S, \theta)$ is then convoluted with the instrument response function to fit the measured profiles. We have tried several distributions including Gaussian, geometric, Raleigh and Gamma distributions, and found that the Gamma distribution has the best fit. The fit of the Raleigh distribution is slightly worse than that of the Gamma distribution. The Gamma distribution has the form:

$$
P(N, \theta)=\frac{1}{\lambda^{\alpha} \Gamma(\alpha)} N^{\alpha-1} e^{-N / \lambda}
$$


with the mean $\overline{\mathrm{N}}=\alpha \lambda$ and distribution width $\sigma \equiv \sqrt{(\mathrm{N}-\overline{\mathrm{N}})^{2}}=\lambda \sqrt{\alpha}$, where $\alpha$ and $\lambda$ were chosen as fitting parameters which are functions of $\theta$ and T. Figure 3(a) shows the angular profiles of the $(1 / 31 / 3)$ beam at different coverages in the [1112] direction from the stepped $\mathrm{Si}(111)$. The solid curves in the figure are the least-square fits obtained utilizing the Gamma distribution in Eq. (2) convoluted with the instrument response function. The best fits at different coverages all give $\alpha=$ $3.3 \pm 0.3$, with $\lambda$ increasing considerably from 7.6 to 23.1 for $\theta \sim 0.05$ to $1 \mathrm{ML}$. The corresponding mean size $\bar{N}$ is calculated to he 24.3 to 69.3 lattice spacings. A similar fit is also obtained in the [211] direction where the hest fit for $\alpha$ is $3.5 \pm 0.3$ and $\lambda$ increases from 10.3 to 28.7 for $\theta \sim 0.05$ to $1 \mathrm{ML}$. The mean size is calculated to be 35.0 to 97.7 . In addition, the width ratio of the $\bar{N}$ in the $[\overline{1} 12]$ to that in th: $[\overline{2} 11]$ is $\sim 0.7$ which is larger than that $(\sim 0.56)$ directly evaluated from the FWHM of the profile. Similar behavior is also found in the flat surface and impurity doped cases. The difference from the FWHM evaluation may be due to the underestimate of the domain size in this incoherent scattering model, especially in the [211] direction in which the profile usually is narrower than in the [112] direction. Shown in Fig. 3(b) and (c) are the evolution of the corresponding size distributions obtained from the best fits. From these figures 'we can gain an insight into three things. First, the dramatic increase of the mean and width in the distribution with coverage indicates that the growth is governed by domain coalescence at the substrate temperature $\mathrm{T}=450^{\circ} \mathrm{C}$. Also, the coalescence occurs more rapidly in the [211] than in the [112] direction due to step effects. Second, when the parameter $\alpha>1$ just as in our case, the Gamma distribution will peak at $N_{p}=(\alpha-1) \lambda$. The steeper slope of the distribution for $N<N_{p}$ than that for $N>N_{p}$ indicates that the probability of finding a domain with size $N$ decreases much faster for $N<N_{p}$ than for $N>N_{p}$ where it decays exponentially. Thus, the interactions among the domain boundaries are strong in the $N$ « $N_{p}$ region and become negligible in the $N$ » $N_{p}$ region. ${ }^{15}$ Therefore, the boundary interactions in our system are a short-range interaction with a range of $\sim \mathrm{N}_{\mathrm{p}} \mathrm{a} .{ }^{15}$ Third, and more interesting, since the growth is governed by dornain coalescence, one expects the existence of scaling, i.e., self-similar growth, in the growth process hecause 
coalescence simply rescales length but leaves the basic morphology of domains unchanged. ${ }^{16}$ Indeed, the Gamma distribution in Eq. (3) \& ith a fixed $\alpha$ can be expressed in a scaling form:

$$
P(N, \theta)=\frac{1}{\alpha \lambda} \frac{\alpha^{\alpha}}{\Gamma(\alpha)}\left(\frac{N}{\alpha \lambda}\right)^{\alpha-1} e^{-\alpha(N / \alpha \lambda)}=\frac{1}{\bar{N}(\theta)} P^{\prime}(x)
$$

where $x=N / \bar{N}(\bar{N}=\alpha \lambda)$ and $P^{\prime}(x)=\left\{\alpha^{\alpha} / \Gamma(\alpha)\right\} x^{\alpha-1} e^{-\alpha x}$ is a scaling function independent of coverage $\theta$. In other words, although the mean size varies with coverage, the functional form of the size distribution does not. According to Eq. (4), we have replotted $\mathrm{P}^{\prime}(x) \equiv \overline{\mathrm{N}} \mathrm{P}(\mathrm{N}, \theta)$ vs $x$ in the inset of Fig. 3(b) and (c). Remarkably, although $P(N, \theta)$ broadens with coverage $\theta$, the $\mathrm{P}^{\prime}(\mathrm{x})$ superpose on each other independent of coverage. This coverage dependent scaling is also observed for the growth on the flat $\operatorname{Si}(111)$ surface. ${ }^{11}$

To confirm the existence of scaling, we have tested it in another way where a power Lorentzian,

$$
I(S)=\frac{A}{\left(S_{\|}^{2}+\kappa^{2}\right)^{m}},
$$

convoluted with the instrument response function is used to fit the angular profiles of the $(1 / 31 / 3)$ beam at different coverages. The inverse correlation length $\kappa$ and exponent $m$ are the fitting parameters, and $\mathrm{A}$ is a constant. We found that Eq. (5) can describe the measured profiles very well with $m=1.5 \pm 0.1$ and $\kappa$ varying with coverage. The fits ${ }^{\prime r}$ shown in the dashed lines in Fig. 3(a) which are essentially indistinguishable from those of Eq. (2) using the Gamma distribution. That the same value of $m$ was obtained for different coverages implies that the angular profile $I(S)$ also contains a scaling function, $I^{\prime}\left(x^{\prime}\right)=1 /\left(1+x^{\prime 2}\right)^{m}$ with $x^{\prime}=S_{\|} / \kappa$. Since the angular profile uniquely reflects the size distribution, it again proves the existence of scaling in the coverage dependent growth. 


\section{Impurity Effects On the $\sqrt{3}$ Domain Crowth}

In the study of impurity effects, the oxygen was chosen as the impurity because oxygen is easily removed to repeat the experiment and is also a common contaminant $\left(\mathrm{SiO}_{2}\right)$ on the $\mathrm{Si}$ surfaces. The experiments were all performed on the stepped Si(111) surface. In the experiment, a small amount $(50.02 \mathrm{ML})$ of oxygen was adsorbed on the clean $\mathrm{Si}(111) 7 \times 7$ at rom temperature before the Ag deposition, and the dose was examined by AES with a relative uncertainty of $\sim 0.3$ $\%$ ML. After the oxygen doping, the 1/7-order beams were almost unchanged. In Fig. 1(a) and (b) the FWHM of the $(1 / 31 / 3)$ beam v's Ag coverage for the two different oxygen doses are show'n in comparison with those for the clean case. As we can see, for any $\theta$ the FWHM broadens with increasing oxygen dose, indicating that the greater the impurity dose, the smaller the $\sqrt{3}$ domains. Also, the ratio of the FWHM in the [211] to that in the [112] increases from $\sim 0.56$ for the pure case. For the oxygen dose of $\sim 0.01 \mathrm{ML}$ the ratio is $0.75 \pm 0.03$ at all $\mathrm{Ag}$ coverages, and for the oxygen dose of $\sim 0.02 \mathrm{ML}$ it varies from $\sim 0.75$ to $\sim 0.88$ for an $\mathrm{Ag}$ coverage of $\theta \sim$ 0.1 to $1 \mathrm{ML}$. Obviously, the impurities make the $\sqrt{3}$ domains nucleate and grow more randomly and isotropically in competition with the step influence. This may be seen more clearly in Fig. 4 where $1 / \mathrm{FWHM}$ vs Ag coverage is plotted in a ln-ln scale for the oxygen dose of $\sim 0.02 \mathrm{ML}$. A power law growth of $\overline{\mathrm{N}}$ with $\mathrm{Ag}$ coverage only holds before $\sim 0.6 \mathrm{ML}$, and after that it almost levels off This indicates that the impurities tend to block the growth when the $\sqrt{3}$ domains grow to a certain size ( 29 lattice spacings fit by Eq. (2) using the Gamma distribution) at the Ag coverage of $\sim 0.6 \mathrm{ML}$. According to Eq. (1) and fitting to the linear slope, the growth exponent $n$ is extracted to be $\sim 0.35$ in the $[\overline{1} \overline{1} 2]$ and $\sim 0.31$ in the $[\overline{2} 11]$ direction. The values of $n$ in the two directions now become closer as compared with those for the stepped surface with no impurities, implying the dominant effects of the impurities over the steps. The slightly smaller value of $n$ in the $[\overline{2} 11]$ direction might be due to the preferential distribution of the oxygen impurities in the $[\overline{2}$ )11] step edge direction. 
In order io see the size distribution in the presence of the oxygen impurities, we have fit Eq. (2), using the Gamma size distribution and convoluted with the instrument response function, to the angular profiles for the oxygen doped cases. Shown in Fig. 5(a) and (b) are the angular profiles in the [ $\overline{1} 12]$ and [ $\overline{2} 11]$ directions, respectively, at different Ag coverages for the oxygen dose of $\sim 0.02 \mathrm{ML}$. The fits (solid curves) are found to be excellent and the best fit size distributions at different coverages are shown in Fig. 6. The corresponding parameters of $\alpha$ and $\lambda$ are $4.4 \pm 0.3$ and 3.7 to 7.6 in the $[\overline{1} 1 \overline{2}]$, and $2.2 \pm 0.2$ and 7.8 to 13.3 in the [211] direction, respectively, for a $\mathrm{Ag}$ coverage of $\sim 0.1$ to $1 \mathrm{ML}$. In contrast to the growth on the clean $\mathrm{Si}(111)$ at the same temperature $\left(\mathrm{T}=450^{\circ} \mathrm{C}\right)$, the size distribution now is much narrower and changes little with coverage, which is quite similar to the growth on the flat and clean $\mathrm{Si}(111)$ at low temperature. ${ }^{11}$ This clearly demonstrates that the impurities will not only make the $\sqrt{3}$ domains nucleate randomly but also tends to prevent domain coalescence and break the long range order in the thin film growth. The difference in $\alpha$ for the two directions means some difference in the size distributions. In the Gamma distribution, a smaller $\alpha$ implies weaker interactions among the domain boundaries. When $\alpha$ reduces to 1 the Gamma distribution becomes the exponential distribution, the continuum limit of the geometric distribution, which implies a totally random distribution of the domain boundaries and a negigible boundary-boundary interaction. ${ }^{15,17}$ Therefore, the smalt $r \alpha$ in the $[\overline{2} 1, j$ may be attributed to more roughening of the boundary structure in this direction than in the [11ㄹㄹ. The impurity-induced roughening at domain boundaries usually will be energetically favored and reduce the boundary-boundary interactions. ${ }^{2}$ Finally, the mean size $\bar{N}$, expressed in lattice spacings, at a Ag coverage from $~ 0.1$ to $1 \mathrm{ML}$ are calculated to be $\sim 16$ to 55 in the $[\overline{2} 11]$ and $\sim 15$ to 50 in the [112] for an oxygen dose of $\sim 0.01$ $\mathrm{ML}$, and $\sim 15$ to 31 in both the directions for an oxygen dose of $\sim 0.02 \mathrm{ML}$. The size decreases with increasing oxygen dose and is much smaller than that grown on the surface with no impurities. 


\section{Conclusions}

We have presented HRLEED data on step and impurity effects on the coverage dependent $\sqrt{3}$ domain growth of $\mathrm{Ag}$ on $\mathrm{Si}(111)$. On the stepped $\mathrm{Si}(111)$, an anisotropic growth and shape in the $\sqrt{3}$ domain are found with the preferential growth along the step edge direction. The growth is governed by domain coalescence at $\mathrm{T} \sim 450^{\circ} \mathrm{C}$ as indicated by the considerable increase in the mean and width of the size distribution, and is self similar (scaling) at different coverages as a result of the coalescence. The size distribution is consistent with a Gamma distribution as determined by a comparison of a sirnple model calculation to the angular profile of a $\sqrt{3}$ diffraction beam. In the presence of oxygen impurities ( $\leq 0.02 \mathrm{ML}$ ), the anisotropy in the $\sqrt{3}$ domain shape diminishes with increasing oxy'gen dose, and the domains nucleate and grow more randomly and isotropically'. Also, the impurities block domain coalescence and break the long range order in the $\sqrt{3}$ structure.

\section{Acknowledgments}

This research was sponsored by the Division of Materials Sciences, U.S. Department of Energy under contract DE-AC.05-84OR21400 with Martin Marietta Energy Systems, Inc. and in part by an appointment to the U.S. Department of Energy Postgraduate Research Program at Oak Ridge National Laboratory administered by Oak Ridge Associated Universities. 


\section{References}

1. B. Lewis and J. C. Anderson, Nucleation and Growth of Thin Films (Academic, New York, 1978).

2. G. S. Grest and D. J. Srolovitz, Phys. Rev. B 32, 3014 (1985); ibid. 3021 (1985); D. A.Huse and C. L. Henley, Phys. Rev. Lett. 54, 2708 (1985); Y. Imry and S.-K. Ma, Phys. Rev. Lett. 35, 1399 (1975).

3. J.-K. Zuo, G.-C. Wang, and T.-M. Lu, Phys. Rev. B 40, 524 (1989).

4. Y. Gotch, S. Ino, and H. Komatsu, J. Cryst. Growth 56, 498 (1982).

5. E. J. Van Loenen, J. E. Demuth, R. M. Tromp, and R. J. Hamers, Phys. Rev. Lett. 58, 373 (1987); R. J. Wilson and S. Chiang, Phys. Rev. Lett. 58, 369 (1987); ibid. 59, 2329 $\left(199^{n}\right.$

6. Y. Horio and A. Ichimiya, Surf. Sci. 164, 589 (1985).

7. T. L. Porter, C. S. Chang, and I. S. I. Tsong, Phys. Rev. Lett. 60, 1739 (1988).

8. M. Saiton, F. Shoji, K. Oura, and T. Hanawa, Surf. Sci. 112, 306 (1981); J.Stohr, R. Jaeger, G. Rossi, T. Kendelewicz, and I. Lindau, Surf. Sci. 134, 813 (1983); S. Kono, K. Higashiyama, and T. Sagawa, Surf. Sci. 165, 21 (1986).

9. E. L. Bullock, G. S. Herman, M. Yamada, D. J. Friedman, and C. S. Fadley, Phys. Rev. B 41, 1703 (1990).

10. Takahashi, S. Nakatani, N. Okamoto, T. Ishikawa, and S. Kikuta, Jpn. J. Appl. Phys. 27, L753 (1988).

11. J.-K. Zuo and J. F. Wendelken, in preparation.

12. U. Scheithauer, G. Mayer, and M. Henzler, Surf. Sci. 178, 441 (1986).

13. J.-K. Zuo, R. A. Harper, and G.-C. Wang, Appl. Phys. Lett. 51, 250 (1987).

14. T.-M. Lu, L.-H. Zhao, M. G. Lagally, G.-C. Wang, and J. E. Houston, Surf. Sci. 122, 519 (1982).

15. J. M. Pimbley and T.-M. Lu, Surf. Sci. 159, 169 (1985); J. Appl. Phys. 58, 2184 (1985).

16. D. Beysens and C. M. Knobler, Phys. Rev. Lett. 57, 1433 (1986).

17. T.-M. Lu and M. G. Lagally, Surf.Sci. 120, 47 (1982); J. M. Pimbley, T.-M. Lu, and G.-C. Wang, Surf. Sci. 159, L467 (1985). 


\section{Figure captions}

Fig. 1 The FWHN of the (1/3 1/3) diffraction beam as a function of Ag coverage (raw data) in

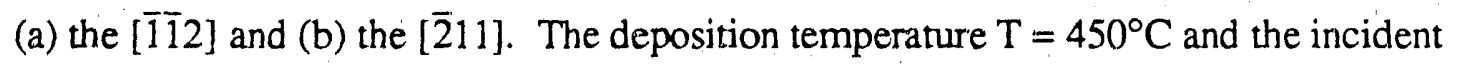
electron energy $\mathrm{E}=84 \mathrm{eV}$.

Fig. 2 The In-ln plot of 1/FWHM vs Ag coverage for the (1/3 1/3) beam for (a) the flat and (b) the stepped $\mathrm{Si}(111)$ surfaces. The deposition temperature $\mathrm{T}=450^{\circ} \mathrm{C}$ and the instrument response width has been removed.

Fig. 3 (a) The angular profiles of the $(1 / 31 / 3)$ beam at different coverages measured from the stepped $\mathrm{Si}(111)$. The solid curves are the best fits of Eq. (2) using the Gamma distribution. The dashed curves are the best fits of the power Lorentzian in Eq. (5) which are nearly indistinguishable from the solid curves. (b) and (c) The evolution of the Gamma size distribution with coverage in the [112] and [211] directions, respectively, obtained using the fitting in (a). The insets in (b) and (c) are the corresponding scaling functions $P^{\prime}(x)$ vs $x$.

Fig. 4 The ln-ln piot of 1/FWHM vs Ag coverage for the (1/3 1/3) beam for the oxygen dose of $\sim 0.02 \mathrm{ML}$. The instrument response width has been removed.

Fig. 5 The angular profiles of the $(1 / 31 / 3)$ beam at different $A g$ coverages in (a) the [1112] and (b) the $[\overline{2} 11]$ directions for the oxygen dose of $\sim 0.02 \mathrm{ML}$. The solid curves are the best fits of Eq. (2) using the Gamma distribution.

Fig. 6 The evolution of the size distribution with Ag coverage obtained by the fits in Fig. 5(a) and (b) for $\mathrm{T}=450^{\circ} \mathrm{C}$. 

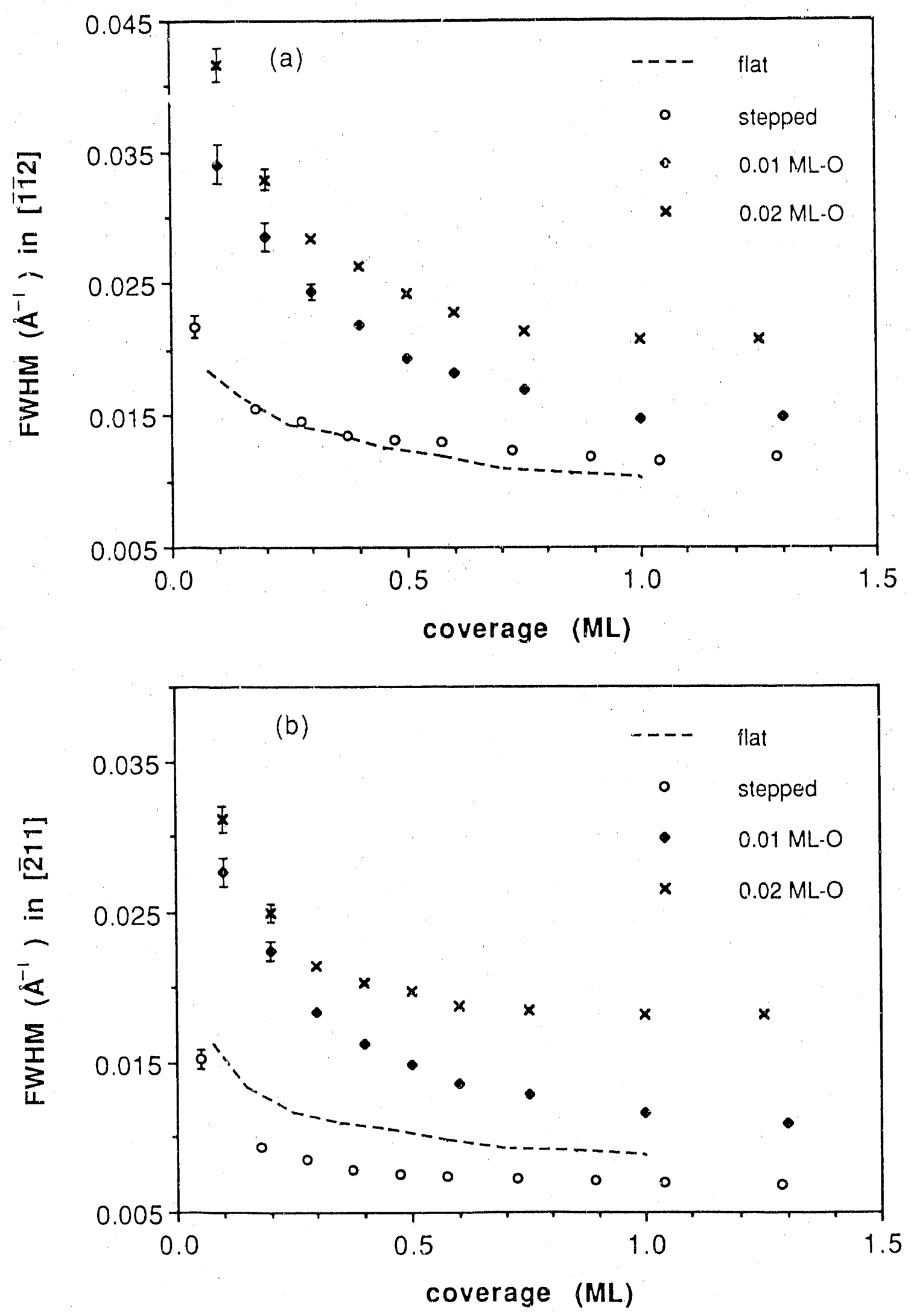

Fig. 1 

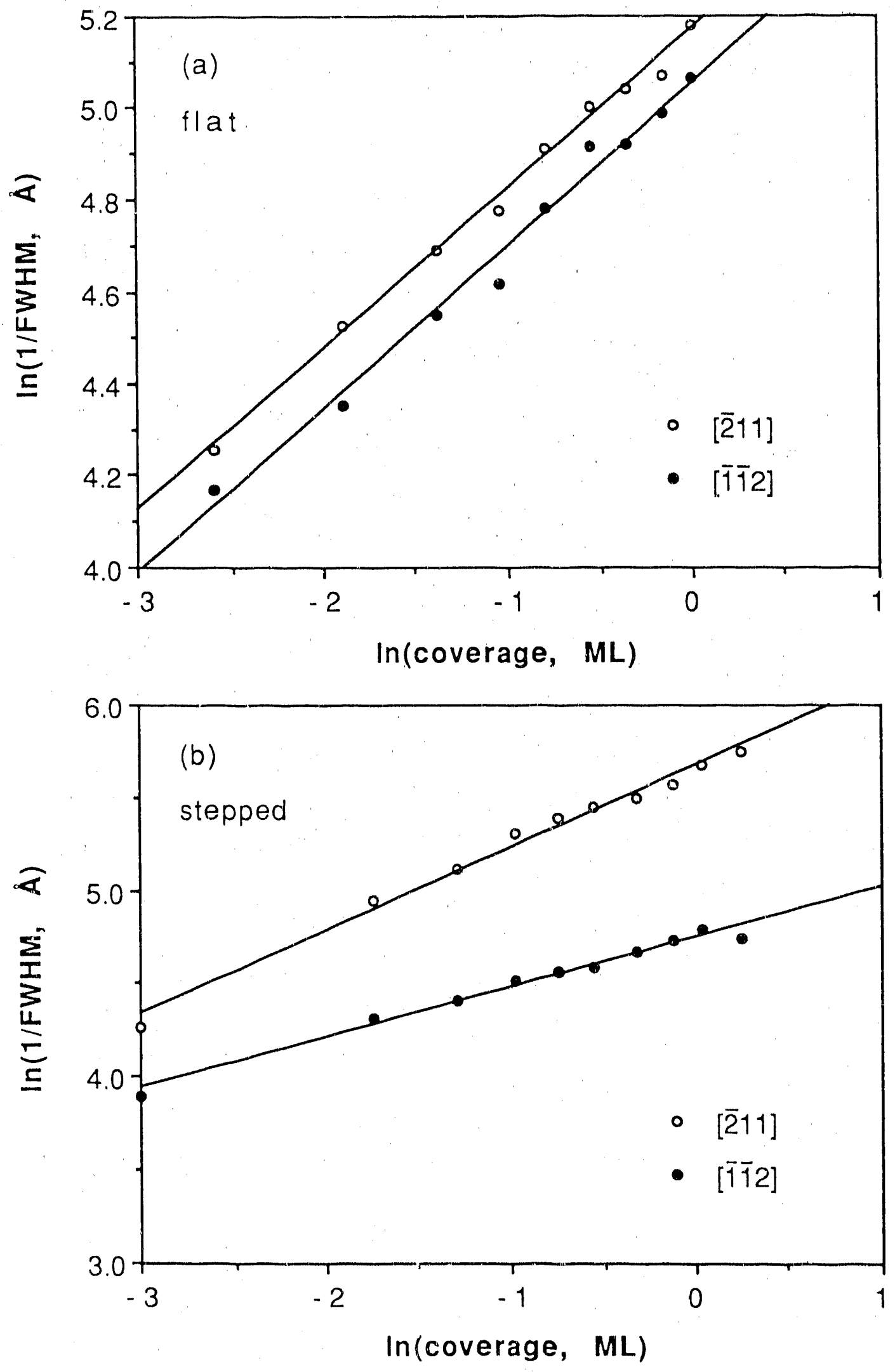

Fig. 2 


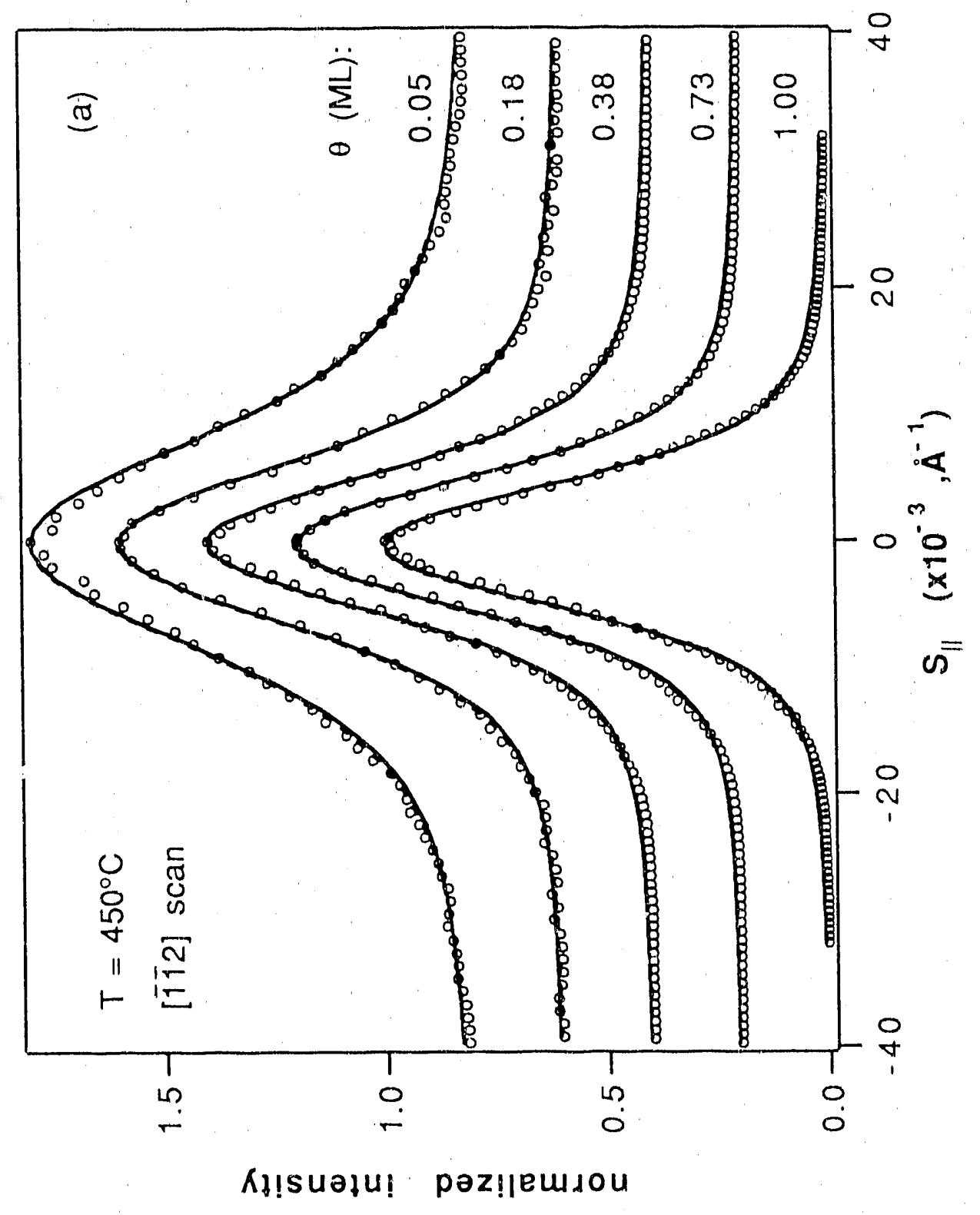




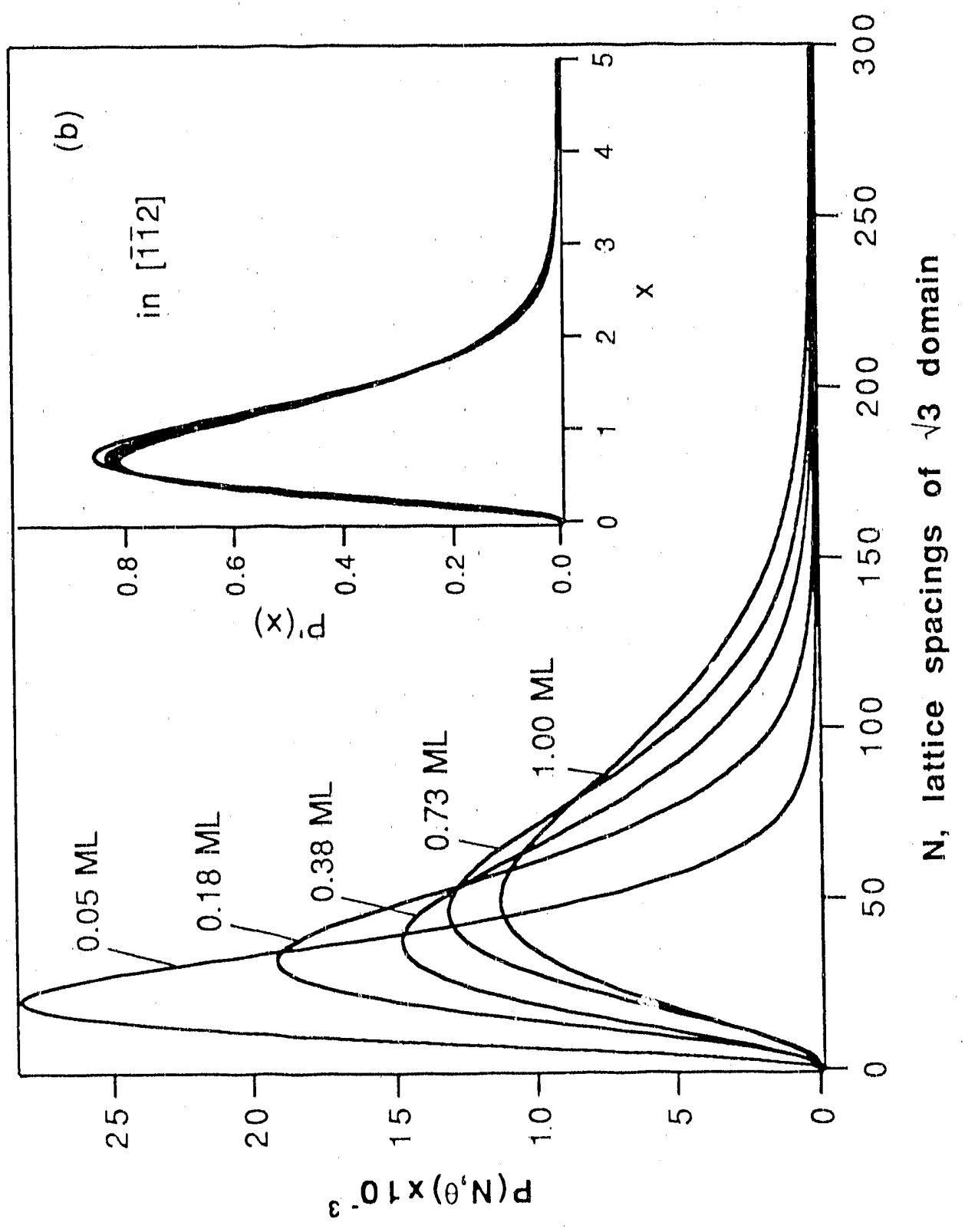




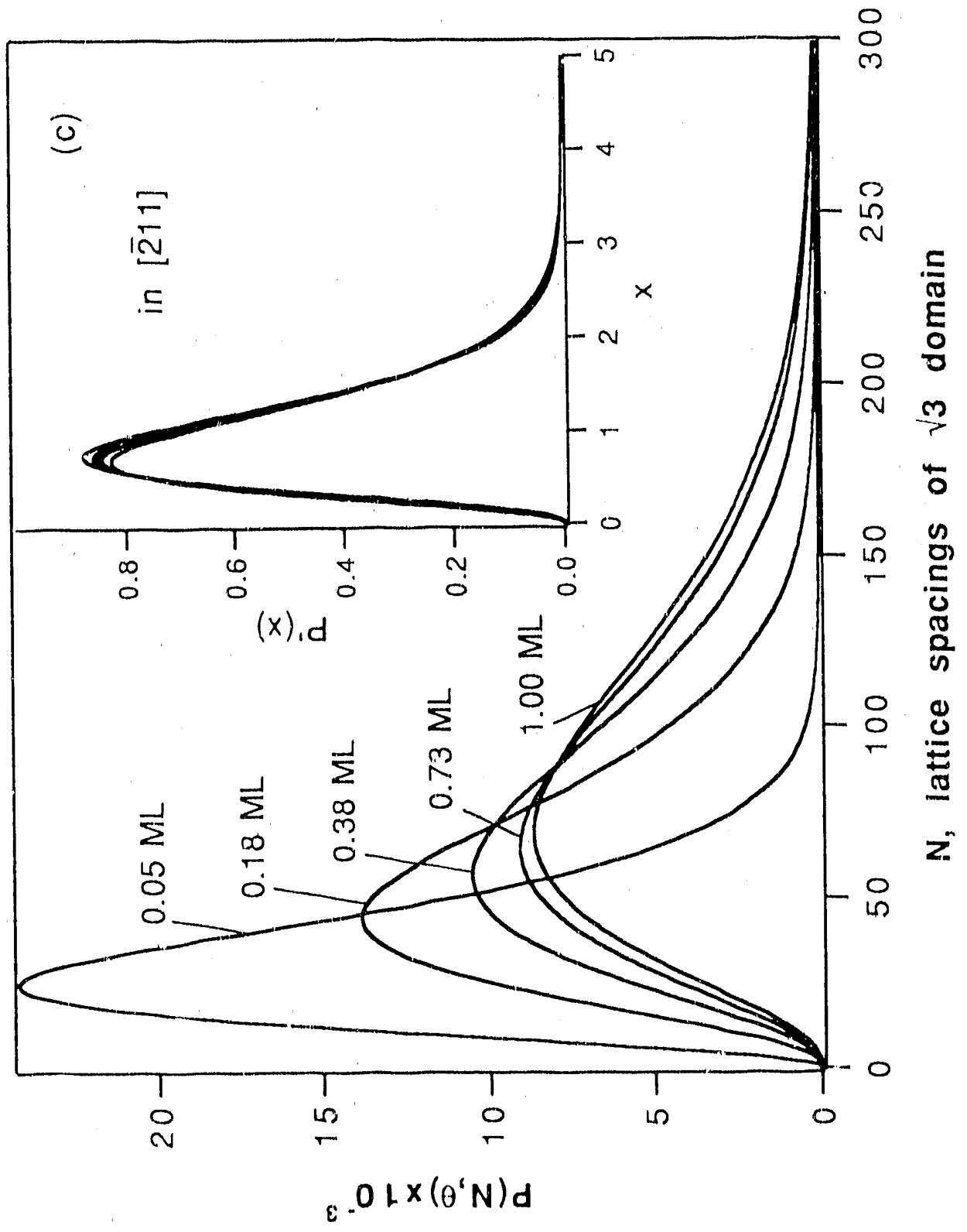




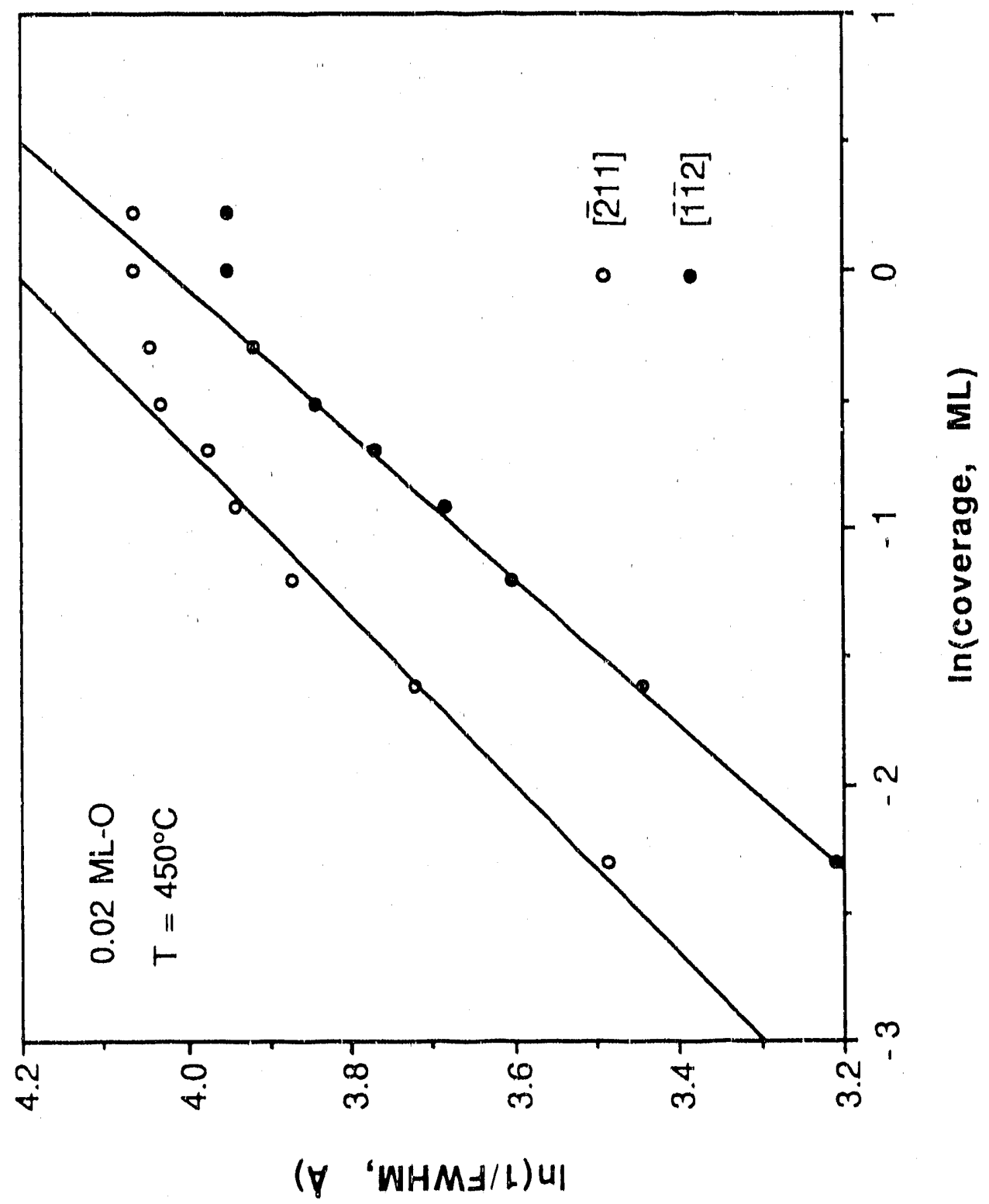




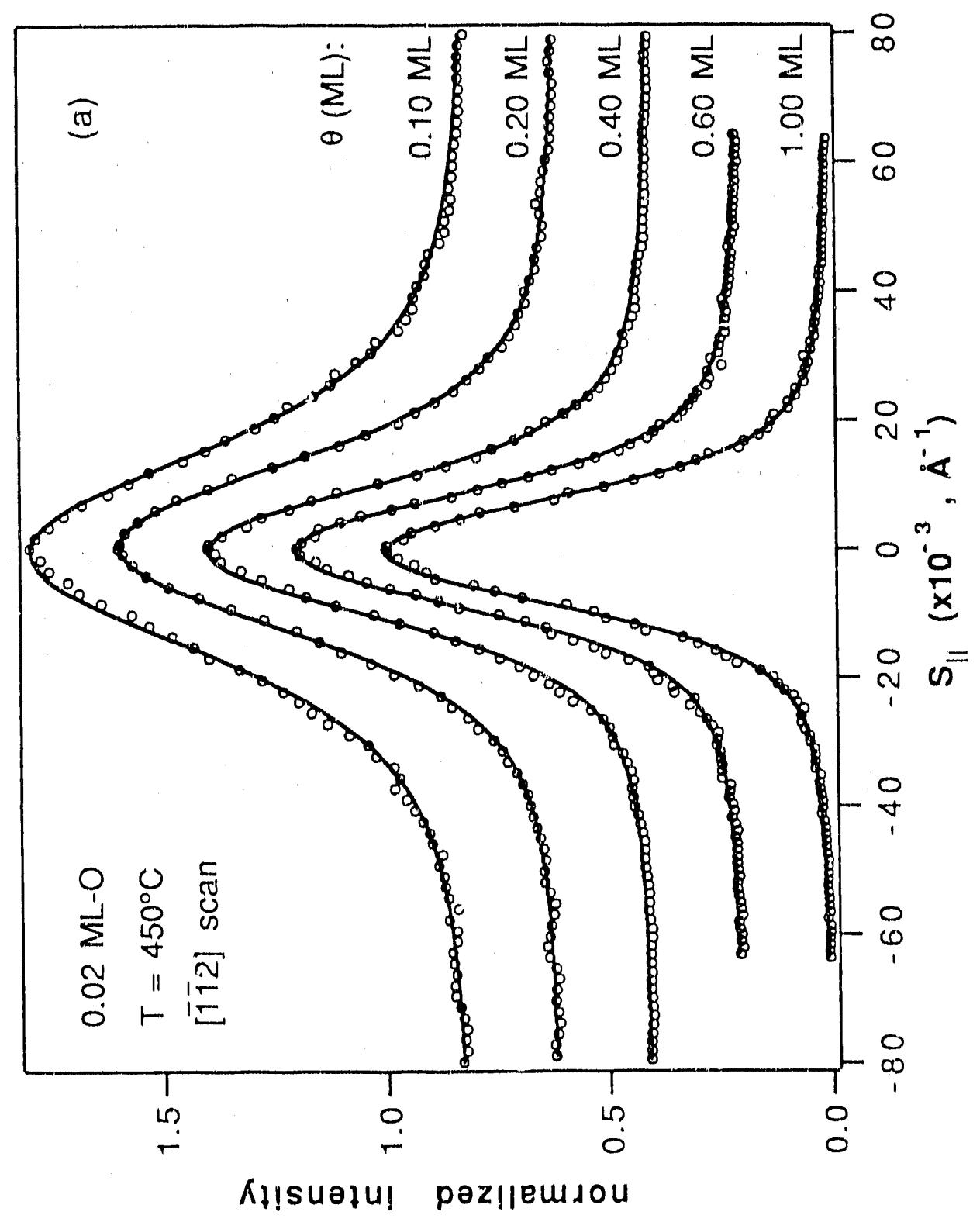




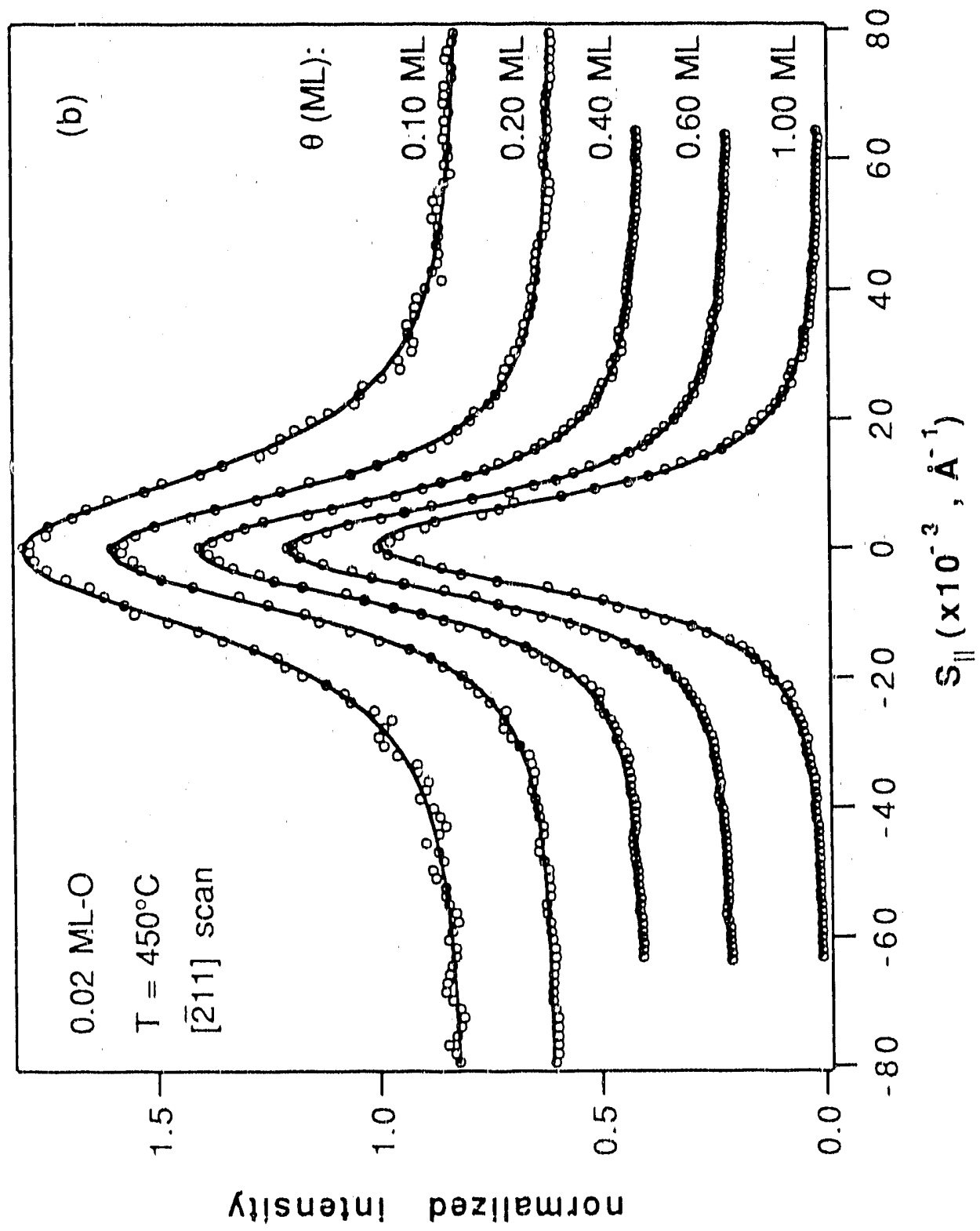




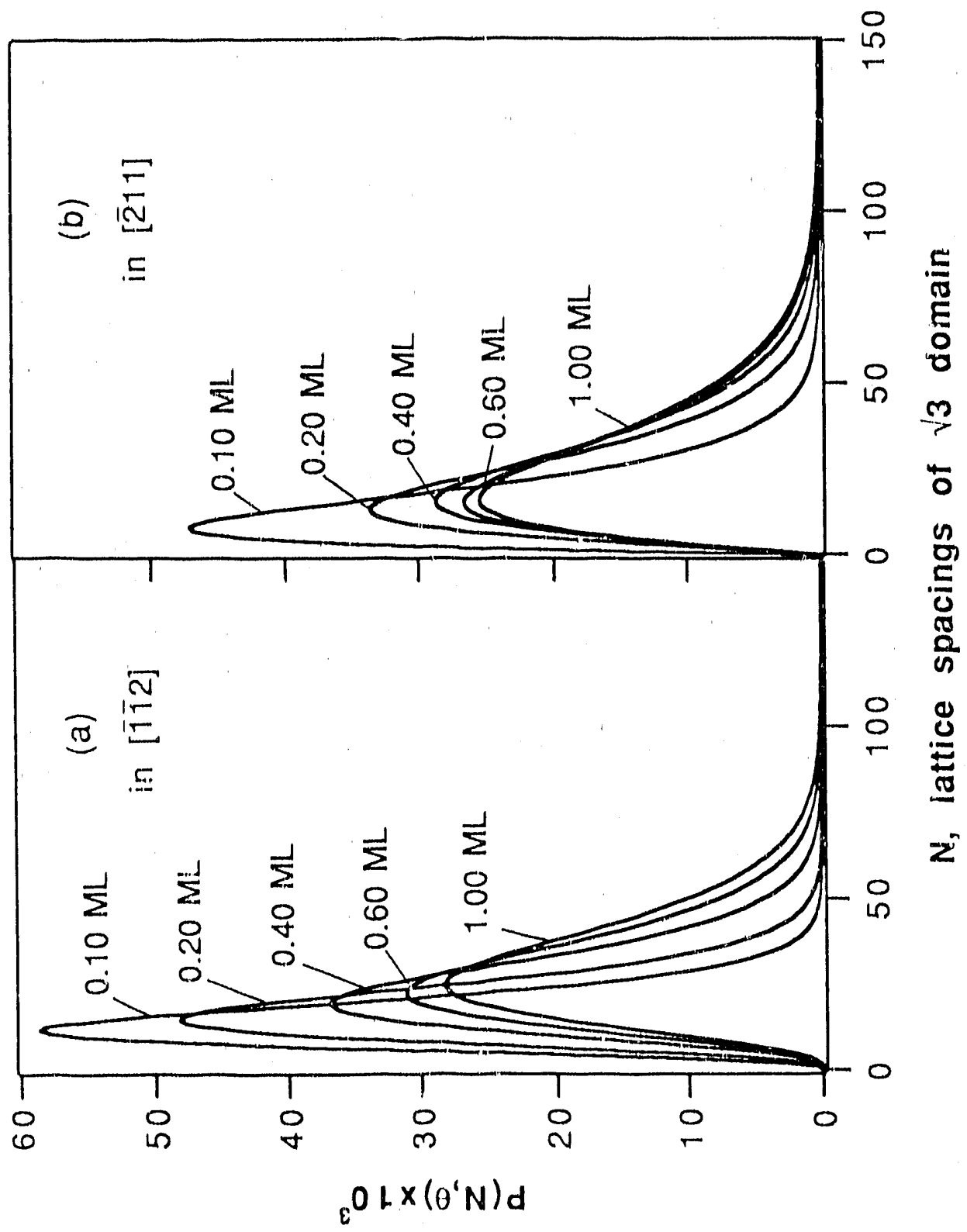



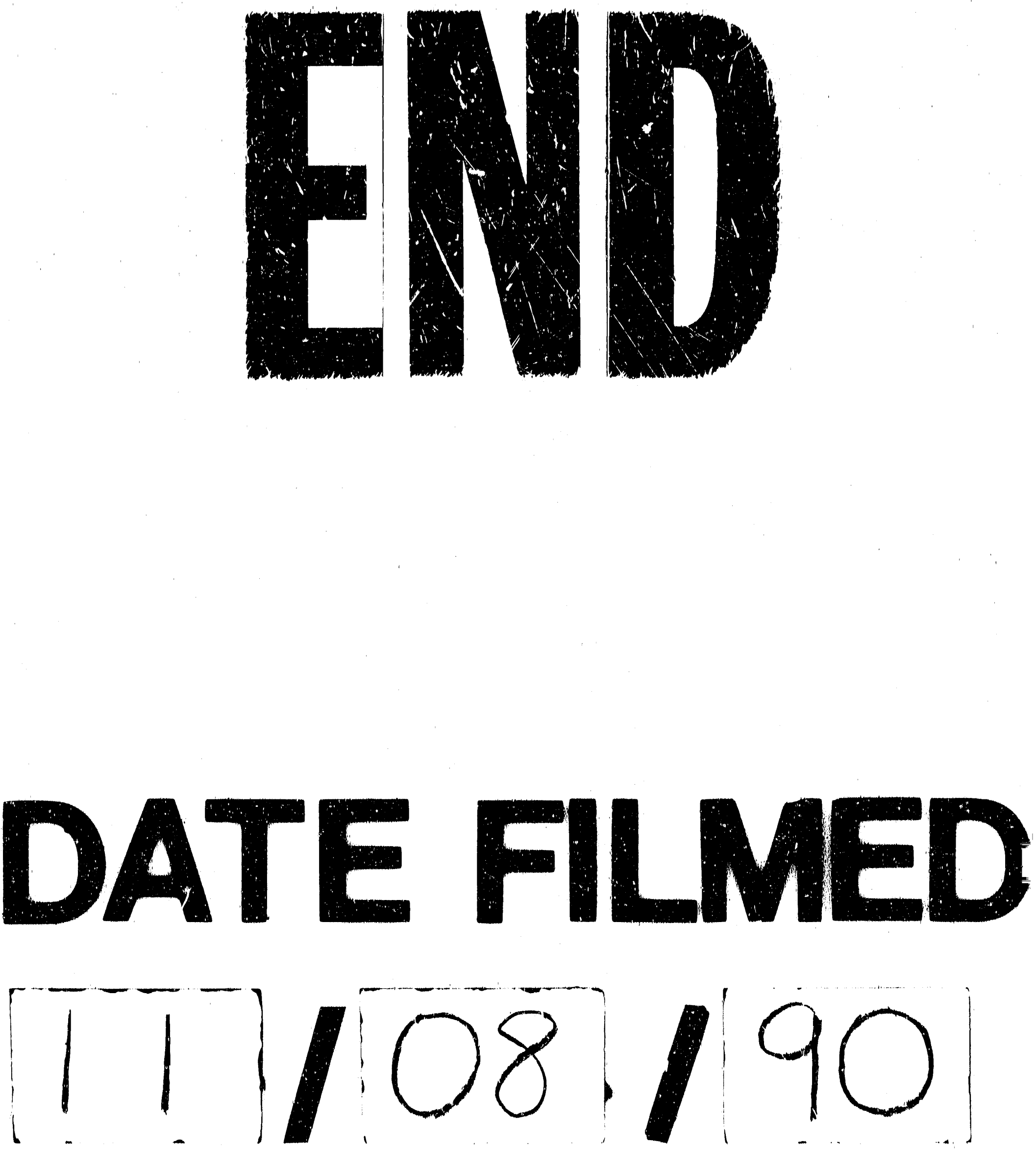
